Louisiana State University

LSU Digital Commons

$1-1-2003$

\title{
Feedforward reduction of the microseism disturbance in a long- base-line interferometric gravitational-wave detector
}

\author{
J. A. Giaime \\ Louisiana State University \\ E. J. Daw \\ Louisiana State University \\ M. Weitz \\ Louisiana State University \\ R. Adhikari \\ LIGO, Massachusetts Institute of Technology \\ P. Fritschel \\ LIGO, Massachusetts Institute of Technology
}

See next page for additional authors

Follow this and additional works at: https://digitalcommons.Isu.edu/physics_astronomy_pubs

\author{
Recommended Citation \\ Giaime, J., Daw, E., Weitz, M., Adhikari, R., Fritschel, P., Abbott, R., Bork, R., \& Heefner, J. (2003). \\ Feedforward reduction of the microseism disturbance in a long-base-line interferometric gravitational- \\ wave detector. Review of Scientific Instruments, 74 (1 I), 218-224. https://doi.org/10.1063/1.1524717
}

This Article is brought to you for free and open access by the Department of Physics \& Astronomy at LSU Digital Commons. It has been accepted for inclusion in Faculty Publications by an authorized administrator of LSU Digital Commons. For more information, please contact ir@lsu.edu. 


\section{Authors}

J. A. Giaime, E. J. Daw, M. Weitz, R. Adhikari, P. Fritschel, R. Abbott, R. Bork, and J. Heefner

This article is available at LSU Digital Commons: https://digitalcommons.Isu.edu/physics_astronomy_pubs/1628 


\title{
Feedforward reduction of the microseism disturbance in a long-base-line interferometric gravitational-wave detector
}

\author{
J. A. Giaime, E. J. Daw, M. Weitz, R. Adhikari, P. Fritschel, R. Abbott, R. Bork, and J. Heefner
}

Citation: Review of Scientific Instruments 74, 218 (2003); doi: 10.1063/1.1524717

View online: https://doi.org/10.1063/1.1524717

View Table of Contents: http://aip.scitation.org/toc/rsi/74/1

Published by the American Institute of Physics

\section{Articles you may be interested in}

Active noise cancellation in a suspended interferometer

Review of Scientific Instruments 83, 024501 (2012); 10.1063/1.3675891

A passive vibration isolation stack for LIGO: Design, modeling, and testing

Review of Scientific Instruments 67, 208 (1996); 10.1063/1.1146573

Multistage active vibration isolation system

Review of Scientific Instruments 69, 2531 (1998); 10.1063/1.1148954

Design of a tuned mass damper for high quality factor suspension modes in Advanced LIGO

Review of Scientific Instruments 88, 035117 (2017); 10.1063/1.4978796

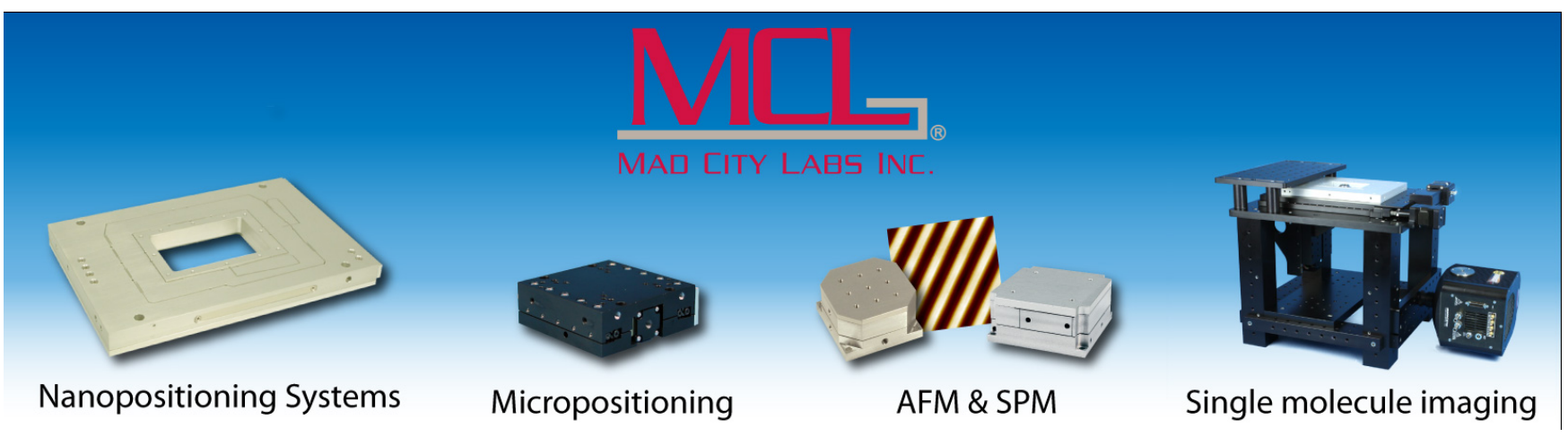




\title{
Feedforward reduction of the microseism disturbance in a long-base-line interferometric gravitational-wave detector
}

\author{
J. A. Giaime, ${ }^{\text {a) }}$ E. J. Daw, and M. Weitz \\ Department of Physics and Astronomy, Louisiana State University, Baton Rouge, Louisiana 70803 \\ R. Adhikari and P. Fritschel \\ LIGO Laboratory, Massachusetts Institute of Technology, NW17-161, Cambridge, Massachusetts 02139 \\ R. Abbott, R. Bork, and J. Heefner \\ LIGO Laboratory, California Institute of Technology, MS 18-34, Pasadena, California 91125
}

(Received 1 August 2002; accepted 25 September 2002)

\begin{abstract}
Standing ocean waves driven by storms can excite surface waves in the ocean floor at twice the wave frequency. These traverse large distances on land and are called the double-frequency (DF) microseism. The Laser Interferometer Gravitational-wave Observatory (LIGO) detector relies on length servos to maintain optical resonance in its $4 \mathrm{~km}$ Fabry-Pérot cavities, which consist of seismically isolated in-vacuum suspended test mass mirrors in three different buildings. Correcting for the DF microseism motion can require tens of micrometers of actuation, a significant fraction of the feedback dynamic range. The LIGO seismic isolation design provides an external fine actuation system (FAS), which allows long-range displacement of the optical tables that support the test mass suspensions. We report on a feedforward control system that uses seismometer signals from each building to produce correction signals, which are applied to the FAS, largely removing the microseism disturbance independently of length control servos. The root-mean-squared displacement from the microseism near $0.15 \mathrm{~Hz}$ can be reduced by $10 \mathrm{~dB}$ on average. (C) 2003 American Institute of Physics. [DOI: 10.1063/1.1524717]
\end{abstract}

\section{INTRODUCTION}

Various research groups around the world are currently commissioning the first generation of long-base-line interferometric gravitational-wave observatories; see Refs. 1 and 2 for recent reviews and references to technical details. The Laser Interferometer Gravitational-wave Observatory (LIGO) consists of $4 \mathrm{~km}$ base-line detectors in Livingston, Louisiana, and Hanford, Washington, and an additional $2 \mathrm{~km}$ detector in Hanford. A simplified detector schematic is shown in Fig. 1. LIGO's operating principle is to use precision laser interferometry to measure the relative motion of seismically isolated test mass mirrors due to the extremely small forces from gravitational waves (GWs) emitted by distant astrophysical events. The test masses are placed at the ends and vertex of an L-shaped vacuum system, in order to be sensitive to the extremely small time-varying quadrupole strain in the gravitational field metric $h \approx 10^{-22}$ that is expected from incident GWs in the $50 \mathrm{~Hz}-5 \mathrm{kHz}$ band.

A Michelson interferometer measures strain $h$ by subtracting the optical phase change that light experiences in traversing one long arm from the change it experiences in the other, perpendicular, arm. The distortion in the local spacetime geometry while a GW passes through the detector is expected to cause an arm-to-arm phase difference that is periodic in the GW frequency. Various optical techniques, including forming resonant Fabry-Pérot cavities in the arms, are used to enhance the interferometric sensitivity to strain

${ }^{a)}$ Electronic mail: giaime@phys.lsu.edu from that of a simple Michelson. Most of the optics and instrumentation are housed in the corner building, with the end test masses in buildings $4 \mathrm{~km}$ away. The entire optical path between the components shown is enclosed within vacuum chambers and pipes.

To achieve the required sensitivity to mirror displacement spectral densities of order $10^{-19} \mathrm{~m} / \sqrt{\mathrm{Hz}}$ at $100 \mathrm{~Hz}$, the LIGO interferometers employ length and angle servocontrol systems to maintain optical resonance in the 4 km FabryPérot arm cavities and in various auxiliary cavities; the Michelson interferometer phase and overall alignment degrees of freedom must also be maintained. ${ }^{3}$ These servos suppress disturbances from, for example, seismic motion with frequencies below the instrument's intended GW detection band and below the effective band of LIGO's seismic isolation system. The controlled degrees of freedom (DOFs) with the greatest required actuation range are the relative displacements between the test masses in the corner station and those $4 \mathrm{~km}$ away in the two end stations. These DOFs are controlled largely by varying electrical current through coils near sets of small magnets that are mounted on the test mass mirrors. To avoid adding unnecessary noise in the detection band, the range of these actuators is quite limited.

Traveling waves in the Earth's surface, which cause vibration in the laboratory floor, can be a source of noise and statistical variation in precision measurements. These effects on sensitive instruments are normally avoided by mounting the instruments on passive or active seismic isolation platforms. Passive systems commonly include air-spring optics table legs and cascaded mass-spring vibration-isolation 


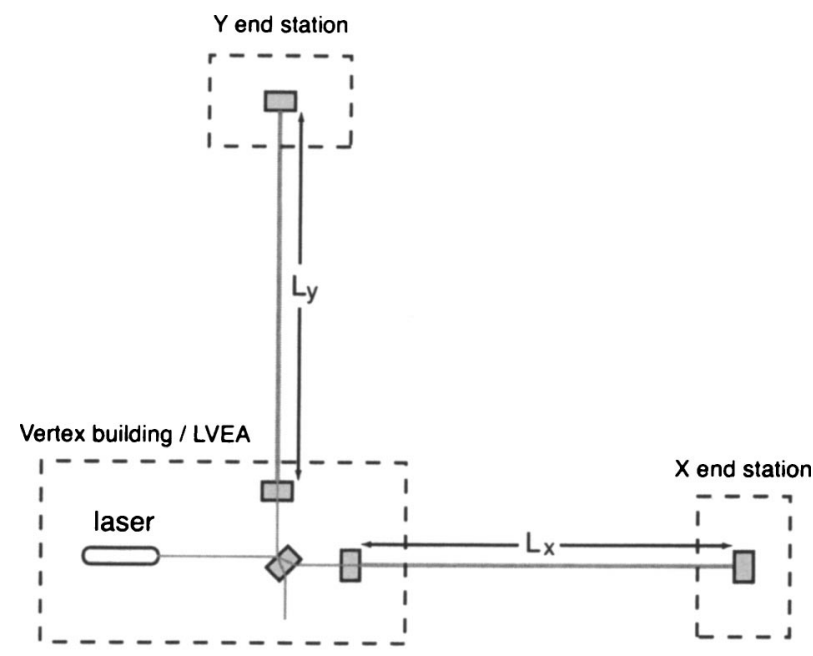

FIG. 1. Sketch of LIGO's arms, simplified here as two perpendicular $4 \mathrm{~km}$ Fabry-Pérot optical cavities combined into a Michelson interferometer.

stacks. ${ }^{4}$ At frequencies above a natural resonance $f_{0}$, each passive stage transmits vibration as approximately $f_{0}^{2} / f^{2}$. Ground-noise displacement isolation factors of $10^{-6}$ have been observed at $100 \mathrm{~Hz}$ in the prototype of the seismic isolation stack used in LIGO. ${ }^{5}$ Another GW detector group uses an ultra-low- $f_{0}$ passive system that can isolate well even as low as several hertz. ${ }^{6}$ Another technique, active seismic isolation, employs force feedback servoloops that use inertial sensors as error signals to quiet a payload in a frequency range where the sensor is quiet and plant dynamics allow sufficient loop gain. An isolation factor of $3 \times 10^{-4}$ has been measured in such a system at $1 \mathrm{~Hz}$ and above. ${ }^{7}$ However, it is difficult to apply either of these techniques to isolate against vibration due to disturbances well below $1 \mathrm{~Hz}$. In the passive case, $f_{0}$ must be significantly below the disturbance frequency. Active feedback isolation is often difficult to make stable at very low frequencies due to the combination of horizontal seismometer sensitivity to payload tilt, and the usual presence of payload dynamics that produce tilt from horizontal force actuation.

A source of low-frequency ground noise that is particularly troublesome to long-base-line interferometric gravitational-wave detectors, and not easily reduced by previously implemented seismic isolation systems, is the double-frequency (DF) secondary microseism. This is produced when standing (counterpropagating) gravity waves in the open sea produce ocean floor pressure variation at double their frequency. The cyclic pressure load excites rapidly moving surface waves in the solid ocean floor, which can then propagate across continents. Reference 8 contains a technical overview with references to research that have led to the current understanding of the sources of both the secondary microseism and the considerably smaller amplitude primary microseism that is observed at the ocean wave fundamental frequency. Commonly measured all over the world, the displacement spectral density peak of the DF microseism is, typically, between 0.1 and $0.3 \mathrm{~Hz}$, with amplitudes ranging from $1 \times 10^{-7}$ to $2 \times 10^{-5} \mathrm{~m} / \sqrt{\mathrm{Hz}}$ at its spectral peak. ${ }^{9}$

At the LIGO Livingston Observatory (LLO) it is not

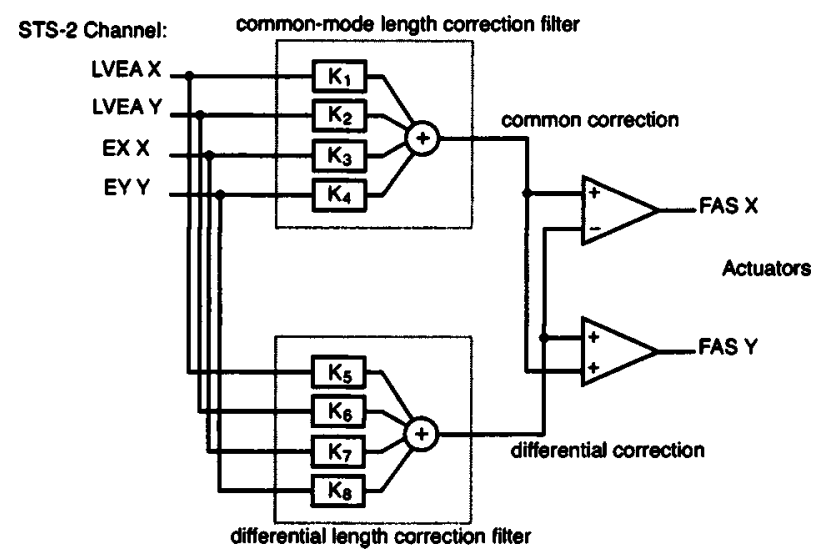

FIG. 2. Block diagram of the feedforward system signal flow from the seismometer channels to the actuators.

uncommon to measure a root-mean-squared (rms) displacement in the microseism band approaching $10 \mu \mathrm{m}$. The DF microseism surface waves travel at about $2 \mathrm{~km} / \mathrm{s}$ with typical wavelengths of about $7 \mathrm{~km}$ at LLO. ${ }^{10}$ The LIGO detector employs length control servos to maintain fixed distances between suspended optics across its two, perpendicular, $4 \mathrm{~km}$ base lines, by applying small control forces directly to the optical elements. These servos then must correct for ground displacements over the base line of the same order as the horizontal microseism ground motion. This would heavily tax the dynamic range of the length control servos.

An alternative active isolation method, called feedforward correction, uses measurements of the ground vibration to predict and cancel the disturbance as transmitted to the test masses. Active feedforward reduction of sound and vibration transmission is a technique that has been studied and used for many decades. ${ }^{11,12}$ Recent papers also indicate its inclusion in GW detector designs. ${ }^{13,14}$ We report here on such a system at LLO, using signals from floor-mounted seismometers to derive length correction information, which is then applied to the seismic isolation system external to the vacuum envelope in order to relieve the test mass actuators of the burden of tracking the microseism in the vicinity of its spectral peak.

\section{SYSTEM DESIGN}

The block diagram in Fig. 2 shows how we have organized the signal flow between the seismometer output channels and the feedforward actuators. The Streckeisen STS-2 seismometers use three internal null-servoed inertial sensors to produce signals along three orthogonal axes. They have a noise floor several orders of magnitude below our typical ground motion at the microseismic peak. One STS-2 is placed in a thermally insulated box on the floor of each LIGO building. Good performance has been obtained by using only four of the seismometer signals, labeled on the left side of Fig. 2; two are in the corner building's laser and vacuum equipment area (LVEA), each aligned with an arm (LVEA X and LVEA Y), and one is in each $X$ and $Y$ end building, aligned with its arm (EX X and EY Y). The filters in the center of Fig. 2 take these inputs and supply predictions of the resulting LIGO arm length changes, which are 


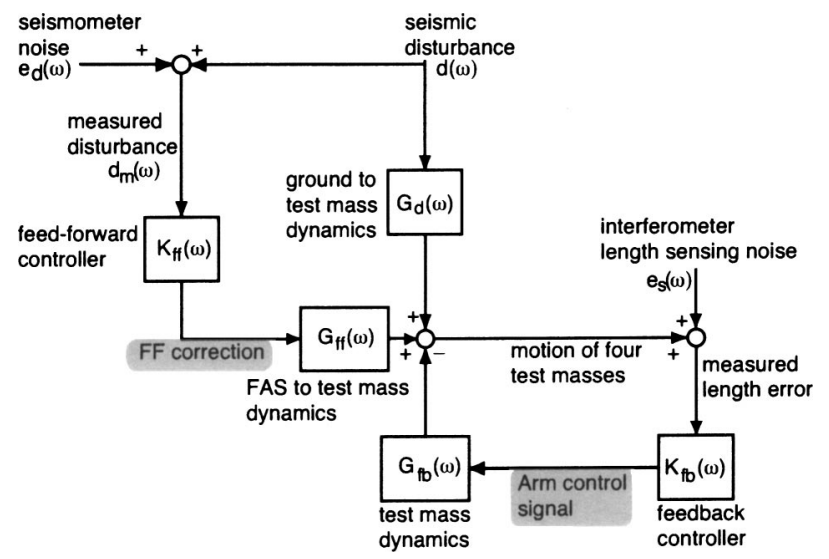

FIG. 3. Block diagram of feedforward and feedback for a single degree of freedom (differential or common mode), showing noise sources and key signals.

then fed forward as shown to the fine actuation system (FAS) inputs in each arm's end test mass seismic isolation system.

The LIGO interferometer measures the deviation away from resonance of its $\mathrm{X}$ and $\mathrm{Y}$ arm cavities in terms of their differential and common-mode length errors. The sum and difference of these signals are applied to the end test mass actuators in feedback loops to maintain arm resonance. With reference to Fig. 1, the common-mode length error is $L_{+}$ $=\left(\delta L_{x}+\delta L_{y}\right) / 2$, where $\delta L_{x}$ and $\delta L_{y}$ are the deviations in arm lengths away from those set by the Fabry-Pérot resonances obtained at initial lock. Similarly, $L_{-}=\left(\delta L_{x}\right.$ $\left.-\delta L_{y}\right) / 2$.

Figure 3 shows both this feedback loop and the microseism feedforward correction path for either the differential or common-mode degree of freedom. The transfer functions shown are all, in general, complex functions of frequency.

In the microseism band, by far the dominant source of test mass disturbance is ground motion, which adds noise to the controlled arm length through the transfer function shown as $G_{d}$. The length errors are sensed interferometrically, using the stabilized laser wavelength as a reference. Then, the length data are filtered by $K_{\mathrm{fb}}$, producing an arm control feedback force that is applied to the test masses. The dynamics of this actuation of the arm lengths is contained in $G_{\mathrm{fb}}$. Feedback reduces the length error by a factor $|S|$, where sensitivity function $S$ is

$$
S=\frac{1}{1+K_{\mathrm{fb}} G_{\mathrm{fb}}} .
$$

In the microseism band, the loop gain $\left|K_{\mathrm{fb}} G_{\mathrm{fb}}\right| \gg 1$, so $|S|$ $\ll 1$ and the feedback loop cancels the disturbance. Each (common-mode and differential) arm control signal can be appropriately calibrated to exactly measure the microseismic motion's effect on the arm lengths, in meters.

To carry out effective feedforward, a system identification (sys-id) process is carried out with the goal of finding the filter $K_{\mathrm{ff}}$ that takes the measured disturbance, $d_{m}$, in four seismometer channels and produces a signal, for which

$$
G_{\mathrm{ff}} K_{\mathrm{ff}} d_{m} \approx-G_{d} d,
$$

applying a correction through the external actuator function $G_{\text {ff }}$ to partially cancel the ground excitation. The presence of noise in $d_{m}=d+e_{d}$ prevents perfect cancellation. There are also other constraints inherent in constructing useful filters, such as the extra phase shifts introduced by bandpassing, which will be discussed in Sec. III.

Disturbance reduction done in this way can benefit the detector while it is acquiring length control servolock, since the interferometer length control signals are not needed. Since feedforward acts outside of servoloops, it can complement LIGO's existing test mass actuated length feedback system, or even a feedback scheme that would allocate some of the actuation to the FAS. Since there is no servoloop, even large system parameter changes would not cause instability or oscillation, but would merely add noise.

\section{A. External actuation of the seismic isolation system}

The possibility of implementing feedforward correction of the microseism was envisioned when the LIGO seismic isolation design was finalized. ${ }^{15}$ The upper line drawing in Fig. 4 shows the seismic isolation overall structure; four piers support a set of external actuators that, in turn, support the in-vacuum four-stage mass-spring passive isolation stack. ${ }^{5}$ The top of the stack supports a "down tube" that ends (hidden from view) as an optics table. The interferometer components, including test mass mirrors suspended from wires as simple pendulums, are attached underneath.

On each pier, the external actuation package, shown enlarged in Fig. 4 (lower detail), includes the FAS, above a vertical scissor jack. The fine actuator supports its payload weight through two flexure plates that have thin sections top and bottom. These allow the payload to swing slightly and be displaced right and left by $\pm 90 \mu \mathrm{m}$. The force to displace the payload is provided by a Polytec PI E-500 series piezoelectric rod actuator linearized by local proportional-integralderivative (PID) servos. One of these is seen shaded and horizontal in the center of the lower drawing, preloaded with coil springs to ensure compressive stress. The FAS components were supplied by Hytec, Inc., Los Alamos, NM.

The actuator rod's internal servo takes as its error signal a co-located strain gauge that responds to the overall rod length, so therefore the payload displacement itself can be "commanded" by the actuator servo input, with very little frequency dependence in its response $G_{\mathrm{ff}}$ within the microseismic band.

\section{B. System identification}

The goal of the sys-id process in this design effort is to obtain a set of linear time-invariant filters that take measured ground motion as input and produce output signals that accurately mimic the LIGO detector's differential and common-mode arm length signals in the microseism frequency band. An important secondary goal is to obtain correction filters $\left(K_{\mathrm{ff}}\right)$ that produce in their outputs very little noise above or below the microseismic peak when using real seismometer data as input. There are two potential sources of noise that are of concern, one at low and one at high frequencies. Very low-frequency floor tilt, produced from time to 


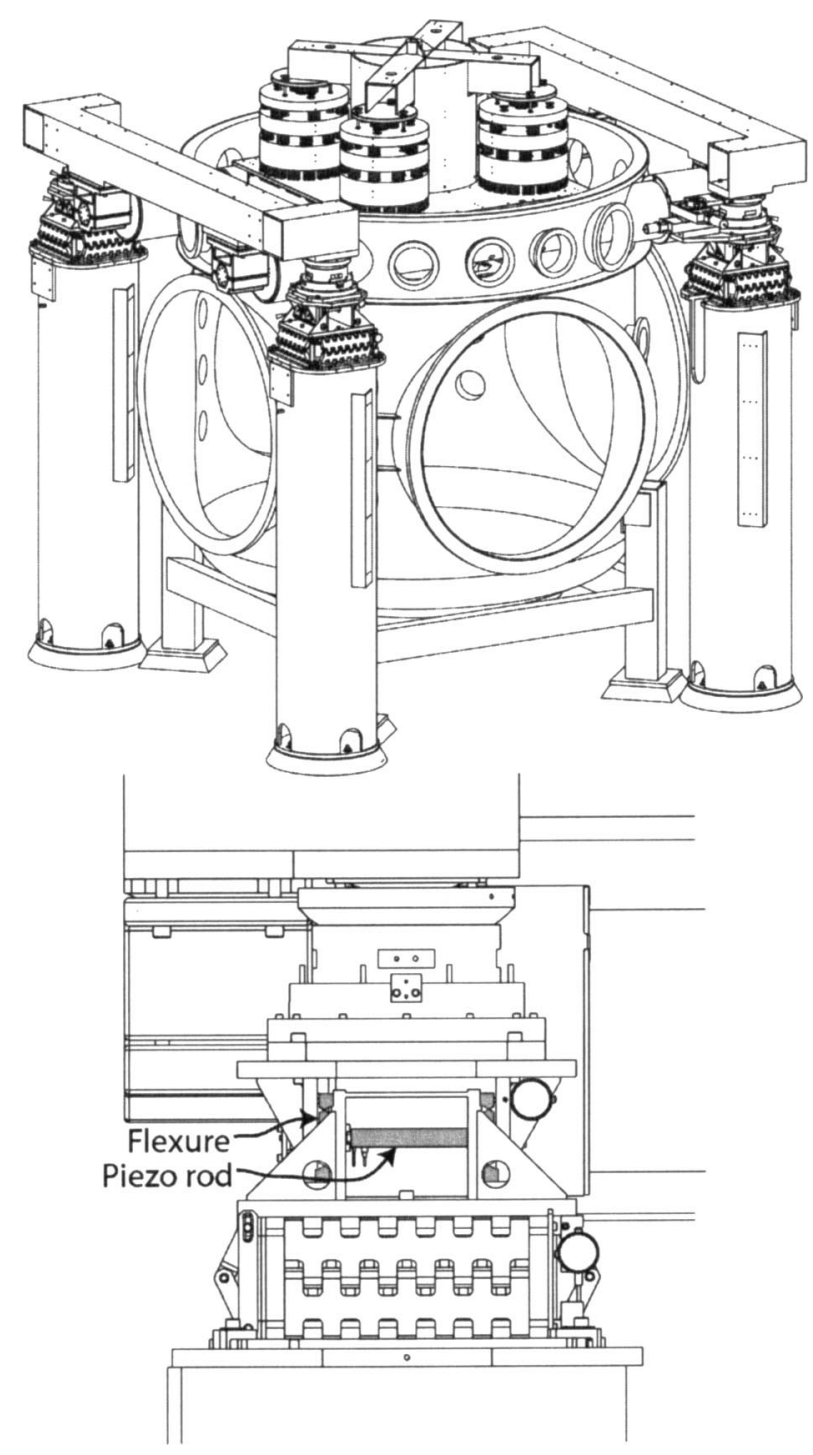

FIG. 4. Drawings of LIGO's seismic isolation system structures. The detail at the bottom shows the external crossbeam supported by a fine actuation system (FAS) consisting of a piezoelectric actuated flexure stage.

time by wind pushing on the building, is seen by the horizontal seismometers as acceleration, and has little predictive power for arm length, so we high-pass filter all of the input channels at $20 \mathrm{mHz}$. High-frequency feedforward actuation must be avoided because the seismic isolation stack has several $Q \approx 30$ resonant peaks in the $1-10 \mathrm{~Hz}$ region that would amplify any filter output in this band. To accomplish this, we low pass the filter input channels at $0.9 \mathrm{~Hz}$.

A black-box system identification tool used with Matlab $^{16,17}$ is used to obtain the differential length error $\left(L_{-}\right)$or common-mode length error $\left(L_{+}\right)$prediction filters by minimizing $\chi^{2}$ between a measured time series for $L_{+}$or $L_{-}$and the output of the filter operating on seismometer ground velocity data. A filter is represented in the form of a discrete-time state space model, ${ }^{18}$ consisting of a set of firstorder linear difference equations that describe the dynamics of a set of state variables, written in matrix form, as well as matrices that link these to the inputs and output. In addition, a noise time series is added to the output.

To construct each $K_{\mathrm{ff}}$, the differential and common- mode feedforward controllers, approximately $200 \mathrm{~s}$ of seismometer and interferometer sensing data at 64 samples/s are collected with the LIGO detector configured to maintain the Fabry-Pérot arm cavity resonance and the Michelson fringe lock, but without "power recycling." ${ }^{3}$ There are three controlled interferometer length DOFs: the Michelson imbalance (an imperfect dark fringe due to motion of the inner test masses with respect to the beam splitter), and the differential and common-mode departures from resonance of the long Fabry-Pérot arms. The Michelson phase error signal is ignored here, since its short base line (several meters) limits sensitivity to the microseism.

Let $\left\{u_{j}(t) \mid j=1 \cdots 4\right\}$ be the input data from the four seismometer channels and $y(t)$ be the real output data, the measured arm control signal. The state space model posits that the input and output data are related through $N_{s}$ state variables $\left\{x_{k}(t) \mid k=1 \cdots N_{s}\right\}$ by

$$
\begin{aligned}
& x_{m}\left(t+\tau_{s}\right)=\sum_{i=1}^{N_{s}} a_{m i} x_{i}(t)+\sum_{j=1}^{4} b_{m j} u_{j}(t), \\
& y(t)=\sum_{k=1}^{N_{s}} c_{k} x_{k}(t)+e(t) .
\end{aligned}
$$

Here, $\tau_{s}$ is the sampling period of the input data. The elements of the matrices $A=a_{m i}, B=b_{m j}$, and $C=c_{k}$ form the space of parameters that can be varied to minimize $\chi^{2}$ between the filter output and the data. The number of these parameters is governed by number $N_{s}$ of the state variables. In addition, the value of the noise time series $e(t)$ is unknown for every time $t$ at which input data are available, and treats the system noise as a single unknown noise source that adds directly to the output $y(t)$.

The sys-id algorithm produces an accurate result for our purposes with ten state variables. The elements of matrices $A, B$, and $C$ are varied without constraint, so there are a total of 150 degrees of freedom for a fit to 13000 data points per measurement channel. A nonlinear Levenberg-Marquardt fit algorithm is employed. ${ }^{19}$ During an iteration of the fit, the input seismometer data are passed through a filter derived from the state space matrices. The difference between the filter output and the measured output data is attributed to the output additive noise $e(t)$. The mean square $e(t)$ over the data sample is the fit discriminant $\lambda$. The fit routine determines the relative magnitudes of the adjustment to the fit parameters based on the first derivatives of $\lambda$ with respect to the matrix elements when far from a minimum (method of steepest descent), and second derivatives when a minimum is approached (the inverse Hessian method). When far from a minimum, the magnitude of the change in fit parameters is halved between successive iterations of the fit routine up to a maximum of 10 . This procedure is repeated, each time using the modified state space as the input guess to the next iteration, until $\lambda$ no longer significantly decreases.

\section{IMPLEMENTATION AND INITIAL TESTING}

Interferometer and seismometer data from the evening of 31 October 2001 were processed by the sys-id procedure outlined above to obtain the feedforward filters for all of the 

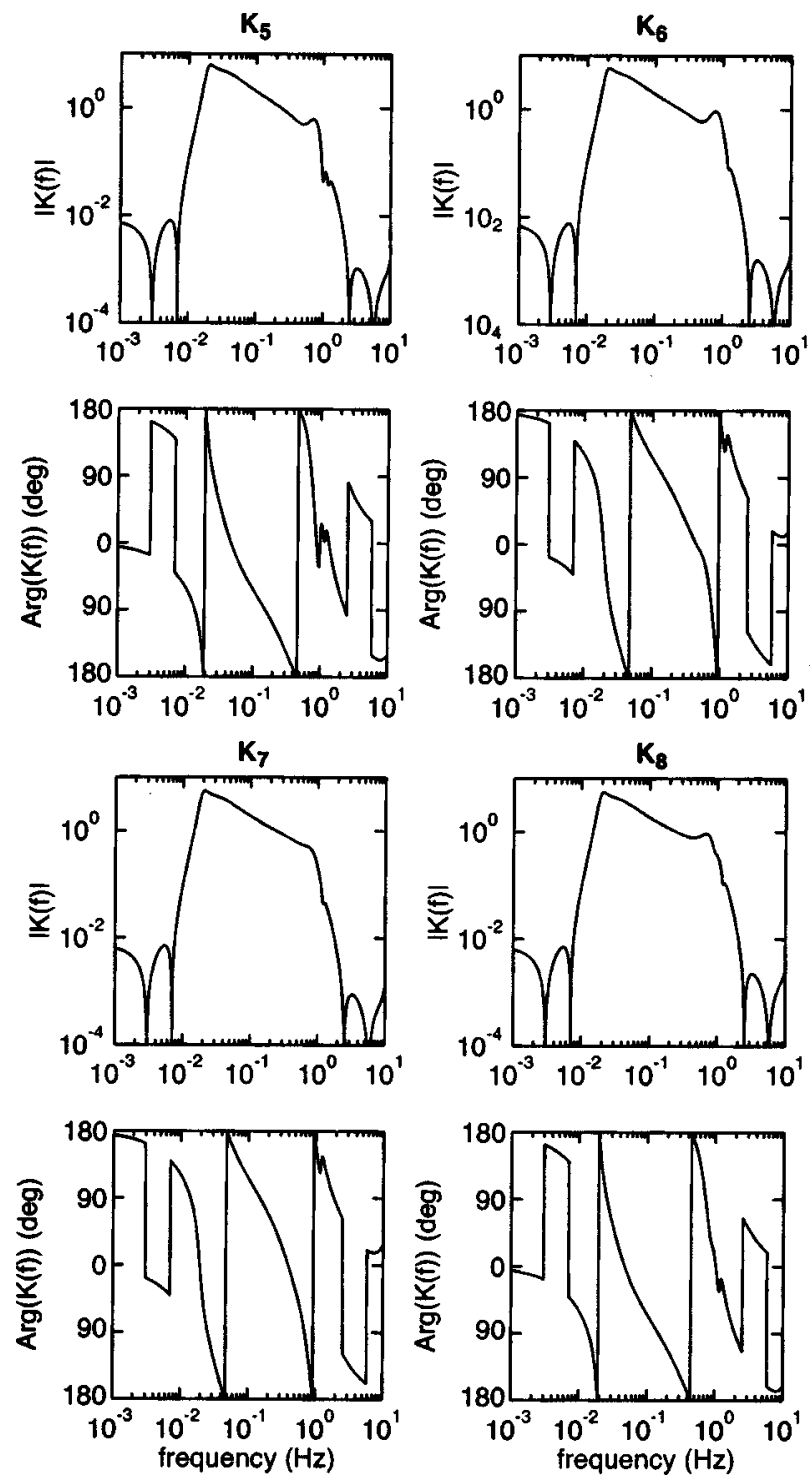

FIG. 5. Filters comprising the differential feedforward correction block.

results described in this article. The filters were converted to the format used in LIGO's digital control processors, and the capability to manually adjust the phase shift near the peak by $\pm 32^{\circ}$ was added. Figure 5 contains transfer function Bode plots of four filters, labeled $K_{5}-K_{8}$ here and in Fig. 3. Each filter takes as input a seismometer channel. The outputs are added together to form the differential arm length error correction signal. Within the bandpass, the magnitude in each falls with frequency, $f$, as $1 / f$, acting at the microseismic peak as an integrator; this transforms the seismometer's velocity output to a displacement readout, which is like the arm control signal. A similar set of filters $\left(K_{1}-K_{4}\right.$ in Fig. 3) comprise the common-mode feedforward controller.

The $20 \mathrm{mHz}-0.9 \mathrm{~Hz}$ bandpass filter, which serves to prevent the feedforward system from adding noise out of its effective band, also limits the system's correction fidelity within its band. The transfer function phase angle of our bandpass varies considerably with frequency, limiting the frequency band over which it can be effective to approximately $0.1-0.2 \mathrm{~Hz}$. It is possible during operation to adjust the "sweet spot" frequency, where the phase and amplitude
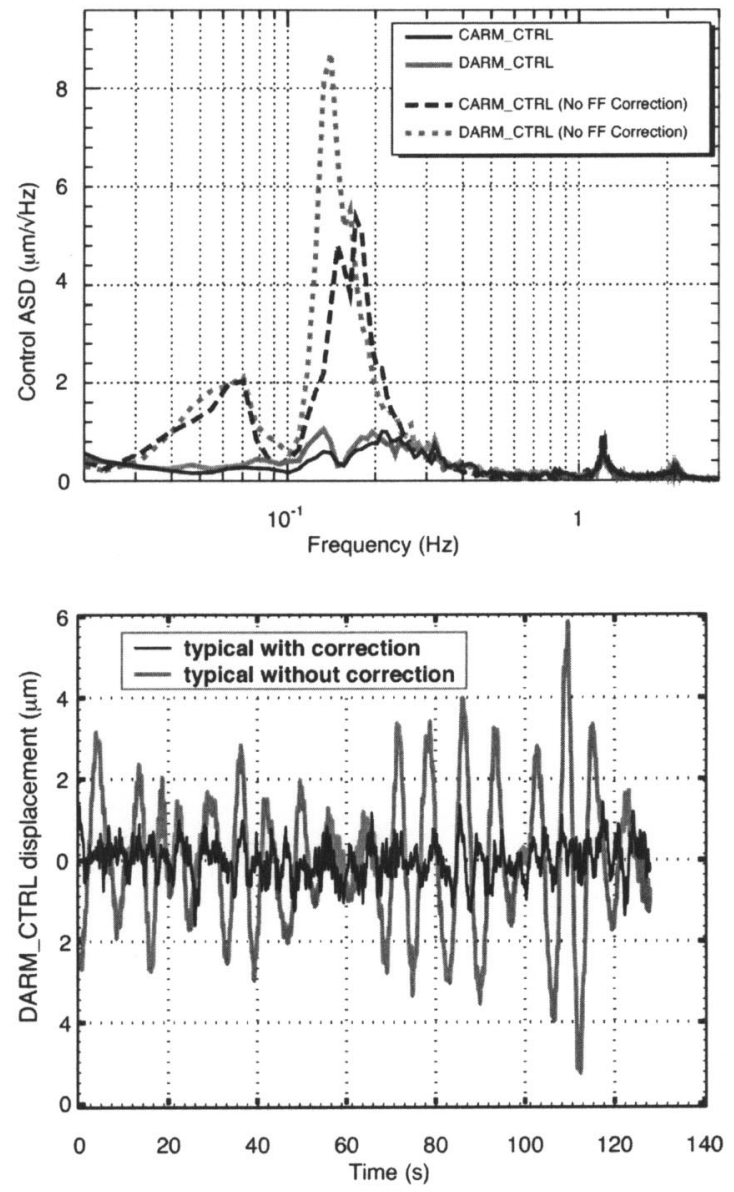

FIG. 6. Upper: the amplitude spectral density of the differential and common-mode arm control signals (DARM_CTRL and CARM_CTRL) calibrated in terms of arm length change, with and without feedforward correction, average over $15 \mathrm{~min}$. Lower: time series segments, with and without feedforward, for DARM_CTRL.

match is nearly perfect, by adding a phase lead or lag filter.

LIGO has been carrying out a series of data-taking runs as part of its commissioning process. Our sixth engineering run (E6), which took place 16-19 November 2001, offered an opportunity to assess the new feedforward system's usability and performance. The DF microseism during this period had its typical spectral shape, strongly peaked at approximately $0.15 \mathrm{~Hz}$ and causing peak-to-peak displacements often approaching $10 \mu \mathrm{m}$ across the LIGO base line. The upper graph in Fig. 6 shows the amplitude spectral density of the arm control signals during two adjacent 15 min periods with the feedforward system on and off. The first $128 \mathrm{~s}$ of each time series from which the differential control PSDs were calculated is shown in the lower graph. It can be seen that the ground excitation was canceled well; the rms differential arm control signal in the $0.03-0.5 \mathrm{~Hz}$ band was reduced $16 \mathrm{~dB}$, and the common-mode control signal by $15 \mathrm{~dB}$.

\section{LONG-TERM TEST}

Between 28 December 2001 and 14 January 2002, a longer engineering run, E7, took place. Over this 432 hour run, the LLO detector's length servos were locked and the 


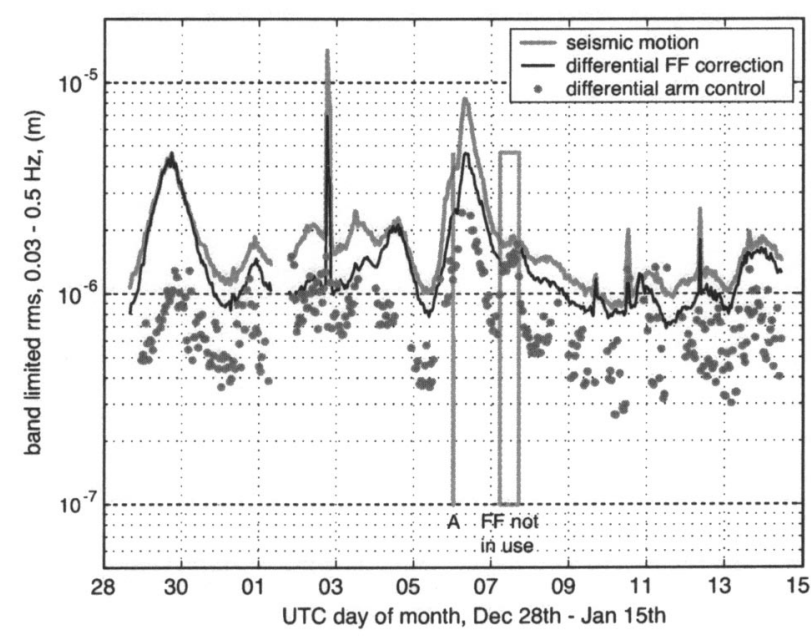

FIG. 7. Comparison of seismic excitation, feedforward correction, and feedback actuation during an 18 day LIGO engineering run. The values shown are the rms displacement in the $0.03-0.5 \mathrm{~Hz}$ band averaged in $50 \mathrm{~min}$ segments. Periods when the LIGO detector's length control system were inoperative are omitted. The half-day-wide gray rectangle highlights a period when the $\mathrm{ff}$ correction was turned off.

instrument was producing clean data for $265 \mathrm{~h}$, a duty cycle of $61 \%$. With the exception of the period UTC 07:13:1816:59:56 on 7, January feedforward was in use for the duration of the run. Comparisons among the interferometer control signals acting directly on the arm mirrors, the feedforward control signals applied through the fine actuators, and a measurement of the horizontal components of the ground velocity (EY Y), are used to monitor feedforward performance over servolocked segments of the run's two weeks.

An off-line analysis pipeline is used to measure the band-limited rms fluctuations of the relevant data channels. Data sampled at $2048 \mathrm{~Hz}$ are read from disk, low-pass filtered, and decimated to a $2 \mathrm{~Hz}$ sampling rate. Two 16th-order digital IIR elliptic bandpass filters are used. One has a $3 \mathrm{~dB}$ passband between 0.03 and $0.5 \mathrm{~Hz}, 0.5 \mathrm{~dB}$ of passband ripple, and $80 \mathrm{~dB}$ of stop-band attenuation. The other bandpass filter has a $0.1-0.2 \mathrm{~Hz}$ passband. The rms variation in each channel, for each bandpass, is calculated and recorded over each 1 min interval over the 18 days of E7. To facilitate comparison among these reduced data channels, all were calibrated in terms of the equivalent (differential and common-mode) arm length change, in meters.

Figure 7 shows the results of the E7 analysis for the differential interferometer degree of freedom and the full $0.03-0.5 \mathrm{~Hz}$ bandpass. The differential feedforward control signal (the solid line) changes the rms arm length difference by up to $5 \mu \mathrm{m}$. It roughly tracks the amplitude of ground motion, as measured in the $\mathrm{Y}$ end building parallel to the arm. On average, the amplitude of the differential arm control signal applied to the test mass mirrors is $5.2 \mathrm{~dB}$ smaller than the feedforward correction we apply. A notable exception is the time period for which the feedforward system was turned off. During this period, the differential arm control signal assumes the level that the microseismic feedforward has immediately before and after the change, which is evi-

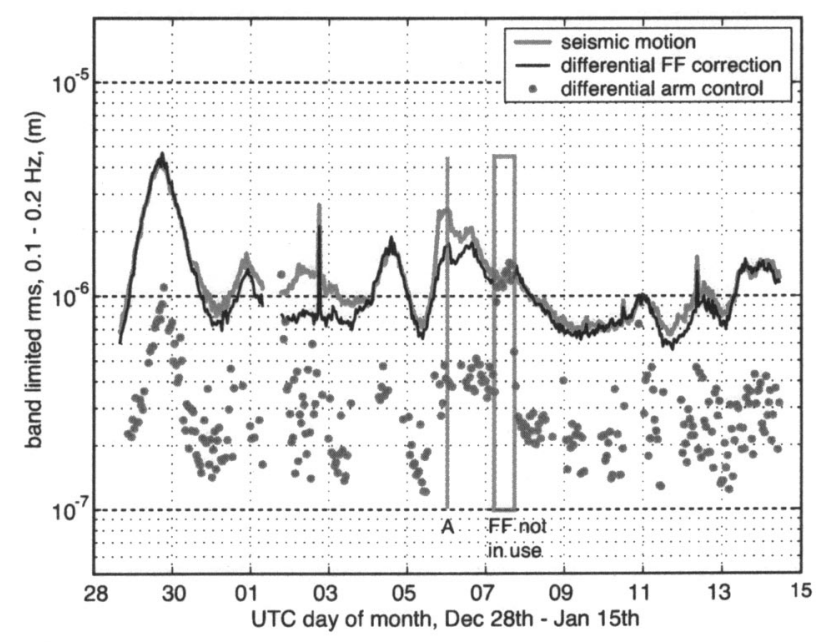

FIG. 8. Comparison of seismic excitation, feedforward correction, and feedback actuation during an 18 day LIGO engineering run. The values shown are the rms displacement in the $0.1-0.2 \mathrm{~Hz}$ band averaged in $50 \mathrm{~min}$ segments. Periods when the LIGO detector's length control system were inoperative are omitted.
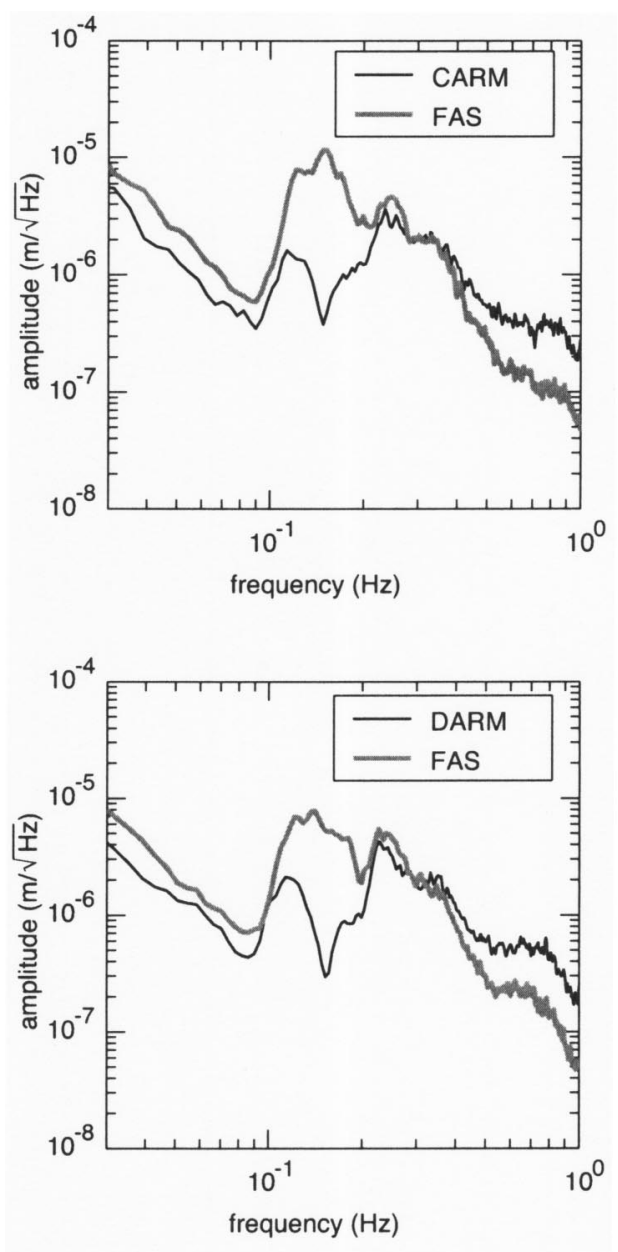

FIG. 9. Amplitude spectral density graphs of the differential and commonmode arm feedback control signals (DARM and CARM), each plotted with its related feedforward correction signal (FAS). These spectra were calculated from data near the time marked "A" in Figs. 7 and 8. 
dence that the broadband rms microseism disturbance is reduced by the feedforward.

Analysis for the narrower, $0.1-0.2 \mathrm{~Hz}$ bandpass filter is plotted in Fig. 8. The feedforward system is reducing the control displacement needed to be applied to the mirrors by an average factor of $10.2 \mathrm{~dB}$ in this band, somewhat better than the wide-bandpass results in Fig. 7. This is because the disturbance in the $0.2-0.5 \mathrm{~Hz}$ band was not suppressed well by feedforward, and during E7 the microseism had a broader peak than usual, with significant power up to $0.3 \mathrm{~Hz}$. This can also be seen in Fig. 9, which shows the amplitude spectral density of the feedforward correction and that of the mirror feedback versus frequency. Note that the disturbance is nicely reduced by feedforward near $0.15 \mathrm{~Hz}$, but that the higher-frequency vibration must be handled by test mass feedback.

\section{DISCUSSION}

We believe that this is the first demonstration of a seismometer-based feedforward system that can significantly reduce relative displacement across kilometer base lines at the double-frequency microseismic spectral peak. The feedforward filters have been effective between 0.1 and $0.2 \mathrm{~Hz}$ for several months after their derivation, regardless of ocean storm conditions that have changed the direction and character of the microseism.

Performance is currently limited above $0.2 \mathrm{~Hz}$ by the severe bandpass that is used to prevent excitation of isolation stack modes. Nonetheless, the overall reduction of the needed feedback range is significant to LIGO operation, since it can allow the use of lower dynamic range actuators and bring a corresponding reduction in system noise that is due to the test mass actuator drive electronics.

We anticipate that a simple external active feedback isolation system (using the same actuation mechanism and an external vibration sensor) can quiet the stack modes and al- low a relaxing of the bandpass cutoff, leading to higherfrequency feedforward performance.

\section{ACKNOWLEDGMENTS}

This work was supported by National Science Foundation Awards Nos. 9801158, 0107417 and 0071316, as well as Louisiana Board of Regents Contract No. LEQSF (2000-03)RD-A-06. The authors also wish to thank the scientific and technical staff of LIGO, and the LSU physics shops.

${ }^{1}$ N. A. Robertson, Class. Quantum Grav. 17, R19 (2000).

${ }^{2}$ J. Hough and S. Rowan, Living Rev. Relativ. 3 (2000), 3. [On-line article]: cited 15 Aug. 2001; http://www.livingreviews.org/Articles/Volume3/20003hough/

${ }^{3}$ P. Fritschel, R. Bork, G. Gonzalez, N. Mavalvala, D. Ouimette, H. S. Rong, D. Sigg, and M. Zucker, Appl. Opt. 40, 4988 (2001).

${ }^{4}$ J. C. Snowdon, Handbook 128 (National Bureau of Standards, Washington, DC, 1979).

${ }^{5}$ J. Giaime, P. Saha, D. Shoemaker, and L. Sievers, Rev. Sci. Instrum. 67, 208 (1996).

${ }^{6}$ G. Ballardin et al., Rev. Sci. Instrum. 72, 3643 (2001).

${ }^{7}$ S. J. Richman, J. A. Giaime, D. B. Newell, R. T. Stebbins, P. L. Bender, and J. E. Faller, Rev. Sci. Instrum. 69, 2531 (1998).

${ }^{8}$ R. K. Cessaro, Bull. Seismol. Soc. Am. 84, 142 (1994).

${ }^{9}$ J. Peterson, Open-file Report No. 93-322, U.S. Department of Interior Geological Survey, Albuquerque, New Mexico (1993).

${ }^{10}$ A. Rohay, Internal Document No. LIGO-C962289-B (1989).

${ }^{11}$ P. Lueg, U.S. Patent No. 2,043,416 (1934).

${ }^{12}$ D. R. Morgan, J. Acoust. Soc. Am. 89, 2362 (1991).

${ }^{13}$ R. Abbott et al., Class. Quantum Grav. 19, 1591 (2002).

${ }^{14}$ B. Willke et al., Class. Quantum Grav. 19, 1377 (2002).

${ }^{15}$ F. Raab and D. Coyne, Internal Document No. LIGO-T960187-01 (1996); http://www.ligo.caltech.edu/docs/T/T960187-01.pdf

${ }^{16}$ L. Ljung, System Identification Theory for the User, 2nd ed. (PrenticeHall, Upper Saddle River, NJ, 1999).

${ }^{17}$ L. Ljung, System Identification Toolbox User's Guide, version 5 (Mathworks, Natick, MA, 2001).

${ }^{18}$ B. Friedland, Control System Design; An Introduction to State-Space Methods (McGraw-Hill, Boston, MA, 1986).

${ }^{19}$ W. H. Press, S. A. Teukolsky, W. Vetterling, and B. P. Flannery, Numerical Recipes in $C$, 2nd ed. (Cambridge University Press, Cambridge, U.K., 1992). 